REVISTA DE LITERATURA E CULTURA RUSSA

Dostoïevski face à l'altérité, entre lecture bakhtinienne et interprétation lévinassienne. Notes sur Dostoïevski, La Question de l'autre, de Jacques Rolland

Dostoevsky and otherness, between Bakhtinian reading and Levinassian interpretation. Notes on Dostoevsky,

La Question de l'autre, de Jacques Rolland

Autor: Yoann Colin Lycée Fénelon,

Paris, Paris, França

Edição: RUS Vol. 12. $\mathrm{N}^{\circ} 20$

Publicação: Dezembro de 2021 DOI: https://doi.org/10.11606/issn.2317-4765.rus.2021.190379 


\section{Dostoïevski face à l’altérité, entre lecture bakhtinienne et interprétation lévinassienne. Notes sur Dostoïevski. La Question de l'autre, de Jacques Rolland.}

Résumé: Il s'agit d'examiner le livre de Jacques Rolland Dostoïevski, la Question de l'autre, parce qu'il propose un éclairage original et fécond sur ce qu'il y a de philosophique dans les grands romans dostoïevskiens, sans sombrer sur l'écueil qui consiste à réduire ces romans à des concepts. Il en ressort une analyse précise de l'altérité, questionnement central dans ces romans, où se mêlent les influences de Levinas, Bakhtine, Catteau et du christianisme.

\begin{abstract}
This article aims at examining Jacques Rolland's book on Dostoevsky and otherness, which shrewdly casts an original light on the philosophical dimension to be found in Dostoevsky's classic novels, without ever subsuming these novels to concepts. As a result, Rolland's analysis puts forward a precise study of otherness - a central question in these novels and influences as diverse as Levinas, Bakhtine, Catteau or Christianism.
\end{abstract}

Mots-clés: Levinas; Bakhtine; Roman; Dostoïevski; Rolland Keywords: Levinas; Bakhtin; Novel; Dostoevsky; Rolland 
* Yoann Colin a poursuivi as formation en classe préparatoire au lycée Fénelon à Paris et a obtenu une équivalence de licence de lettres modernes et licence de philosophie. II a ensuite obtenu une maitrise et un master 2 en philosophie à la Sorbonne. II enseigne la philosophie au lycée depuis 2007. II a obtenu en 2011 une licence de théologie catholique à Strasbourg, où il a soutenu sa thèse de philosophie en 2020; yoanncolin_538@hotmail.com

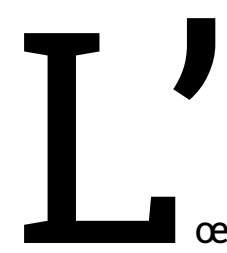

œuvre de Dostoïevski a influencé considérablement un grand nombre de penseurs et un examen de cette influence requerrait un travail d'une immense ampleur. C'est pourquoi plusieurs tentatives pour rendre compte de ce qu'on a pu appeler la " philosophie » de Dostoïevski ont vu le jour. Ces tentatives ont certes le but théorique louable de vouloir traduire en concepts ou en système ce que les écrits de Dostoïevski, et particulièrement ses grands romans, donneraient à penser. Or, dans Dostoïevski. La Question de l'autre, Jacques Rolland s'emploie à la fois à récuser de telles tentatives en en montrant la structurelle insuffisance et en soulignant l'hétérogénéité des romans dostoïevskiens et de l'approche philosophique, et à proposer une réflexion plus qui a plus humblement pour thème le roman dostoïevskien, en s'inspirant notamment des pensées croisées de Levinas et de Bakhtine.

\section{L'œuvre romanesque de Dostoïevski : une singularité irréductible au concept}

Dans Dostoïevski. La Question de l'autre, Jacques Rolland vise une «approche philosophique de l'œuvre littéraire de Dostoïevski», littéraire car l'analyse se fonde essentiellement sur l'œuvre romanesque et car il s'agit de prendre «l'œuvre telle qu'en elle-même elle est et se donne pour en assumer le caractère non-philosophique dont la reconnaissance et l'affirmation nous semblent la prémisse de toute tentative d'approche». ${ }^{1}$ Le geste méthodique consiste en un mouvement de la philosophie vers l'œuvre littéraire, et non pas en un trans-

1 Rolland, 1983, p. 15. 
port de l'œuvre littéraire dans «le champ de la philosophie». ${ }^{2}$ Néanmoins, si le matériau sur lequel s'exerce sa lecture est romanesque, et donc littéraire, Rolland procède à une Lecture philosophique, et non psychologique, psychanalytique, sociologique, historique ou stylistique. Il s'agit bien, en quelque sorte, d'examiner un champ de problèmes philosophiques et de voir comment l'œuvre de Dostoïevski peut nous permettre d'y répondre, même s'il ne le fait pas directement et dans un langage philosophique en tant que tel.

Ce qui justifie qu'on n'interroge pas l'œuvre de Dostoïevski comme si elle était intrinsèquement philosophique, c'est qu'une telle interrogation ne pourrait que méconnaître et réduire la portée de l'œuvre et ne serait qu'une présentation déformée et insatisfaisante de ce que serait prétendument la pensée de l'auteur. Et Rolland se justifier en présentant - et en la critiquant - l'interprétation de Berdiaev. Ce dernier en effet sépare l'œuvre de Dostoïevski en deux et en rejette ce qu'il appelle des "œuvres de jeunesses», et considère comme non essentielle la question de la pauvreté et de l'humiliation. ${ }^{3}$ L'interprétation de Berdiaev, note-t-il,

mutile encore intérieurement ce qu'elle présente pourtant comme son noyau authentique en sous-entendant que la dimension de la pauvreté, de l'humiliation ou de l'offense en serait absente. Il importe au contraire de bien comprendre que cette dimension ne cesse de marquer la totalité de l'œuvre et affirme sa présence jusque dans le dernier roman publié. 4

Ainsi Berdiaev développe une interprétation qui réduit préalablement l'œuvre de Dostoïevski. C'est une violence contre l'œuvre de Dostoïevski, violence qui se retrouve toujours lorsqu'on fait de Dostoïevski un philosophe.

Aussi fait-il en revanche droit à l'approche de Bakhtine qui ne construit plus une "philosophie de Dostoïevski» en identifiant tel discours ou le trajet de tel personnage qu'on considère comme porte-parole de la pensée de Dostoïevski. Pour

2 lbid.

3 Berdaiev,1974.

4 Rolland, 1983, p. 17. 
Bakhtine, le cœur de l'œuvre de Dostoïevski est une structure: le principe polyphonique. C'est moins le contenu thétique de l'œuvre que ce dernier essaierait de repérer que ce sa forme pourrait avoir à signifier.

Par sa définition même, la polyphonie implique la co-existence d'une multiplicité de voix indépendantes les unes par rapport aux autres et, par ailleurs, douées d'une certaine liberté au regard de l'auteur. Dans le roman polyphonique, les voix sont des personnages, lesquels se définissent avant tout par leur parole. Parole sur le monde et parole sur eux-mêmes [...] du fait du principe d'indépendance des voix qui régit la polyphonie, «les héros de Dostoïevski, tels que les conçoit l'écrivain lui-même, ne sont pas seulement produits de la parole de l'auteur mais aussi sujets de leur propre parole directement signifiante». ${ }^{5}$

Cette conception dialogique n'est pas sans rappeler les analyses développées par Buber ${ }^{6}$ et Levinas, ${ }^{7}$ qui insistent sur l'hétérogénéité irréductible des singularités les unes par rapport aux autres. Autrement dit, Pour Bakhtine, selon Rolland, ce qui est central dans les romans de Dostoïevski, c'est leur

5 Ibid., p. 19. II synthétise ainsi l'analyse de Bakhtine: «Polyphonie romanesque, l'œuvre de Dostoïevski prend ainsi, considérée dans sa totalité, l'aspect d'un grand dialogue. Dialogue dans lequel les deux logoi conservent chacun sa personnalité et ne se fondent jamais dans l'unité. Dialogue dans lequel l'interlocuteur n'est jamais seulement un ceci ou un cela, un quelque chose - dialogue dans lequel toujours il est d'abord un Tu ou un Toi, c'est-à-dire quelqu'un à qui je dois m'adresser et qui peut me répondre.» (Ibid., p. 23). Bakhtine écrit que dans les romans de Dostoïevski «c'est précisément une pluralité de consciences, ayant des droits égaux, possédant chacune son monde qui se combinent dans l'unité d'un événement, sans pour autant se confondre. [...] La conscience du personnage est donnée comme une conscience autre, comme appartenant à autrui, sans être pourtant réifiée, refermée, sans devenir le simple objet de la conscience de l'auteur [...] Dans ses œuvres apparaît un personnage dont la voix est construite comme l'est, dans un roman de type ordinaire, la voix de l'auteur même, non celle de son personnage. Le discours que le personnage tient sur le monde a le même poids que le discours d'un auteur ordinaire; il n'est pas soumis à l'image objectale du personnage, comme l'une de ses caractéristiques; mais il ne sert pas non plus de porte-voix à l'auteur. II possède une exceptionnelle indépendance dans la structure de l'œuvre, il résonne en quelque sorte aux côtés du discours de l'auteur, et se combine d'une façon particulière avec lui ainsi qu'avec les voix également qualifiées des autres personnages» (cité par Todorov, 1981, p. 161-162). II note également: «Après Dostoïevski [...] est apparu le rôle d'autrui, à la lumière duquel se construit tout discours sur soi» (cité ibid., p. 163). L'idée du personnage chez Dostoïevski renforce la consistance du personnage face à l'extériorité à laquelle il est confronté (Bakhtine, 1970, pp. 125-143).

6 Que connaissait Bakhtine, comme le rappelle Rolland s'appuyant sur la lecture de Todorov, 1981, p. 151, note 1.

7 Que cite Rolland, 1983, p. 23. 
structure, dialogique, qui fait droit à la singularité et à la relative autonomie de chaque personnage, et non à la subordination des personnages secondaires au développement des convictions qui seraient celles du personnage principal, porte-parole de l'auteur. Et puisque ce qui est premier c'est l'indépendance de personnages consistants les uns par rapport aux autres et aux visées de l'auteur, chaque personnage se construit nécessairement à travers ses interactions et ses réactions par rapport aux autres. C'est pourquoi la question philosophique centrale qui se déduit de l'analyse basée sur la forme des romans dostoïevskiens, c'est la «question de l'Autre», la «question du rapport à autrui». ${ }^{8}$ Autrement dit, suivant l'analyse de Bakhtine, Rolland lit l'œuvre de Dostoïevski comme la «tentative d'une approche philosophique de Dostoïevski prenant pour fil conducteur la question de l'Autre». ${ }^{9}$

\section{Un Dostoïevski lévinassien}

Ce qu'offre d'original l'analyse de Rolland c'est qu'elle ne se penche pas sur l'influence de Dostoïevski sur Levinas (influence d'ailleurs avouée et presque revendiquée), mais elle rend compte des mouvements romanesques dostoïevskiens en langage, en termes, en concepts lévinassiens, comme si, Éveilà rebours de toute chronologie, l'attitude de certains personnages des grands romans de Dostoïevski incarnaient, manifestaient, donnaient chair à des concepts développés dans la philosophie d'Emmanuel Levinas. Il faut même souligner que la réflexion de l'auteur sur Dostoïevski emprunte plus à Levinas qu'aux autres commentateurs. L'intérêt de cette quasi-reformulation dans le lexique de Levinas d'épisodes de grands romans est double. D'une part, ce travail permet de rendre compte philosophiquement de ce qui advient littérairement sous la plume de l'illustre romancier russe, d'autre part, il situe

$8 \mathrm{lbid}$. p. 29

$9 \mathrm{lbid}$. 
de façon décisive le questionnement philosophique porté par ces romans dans le champ problématique de l'altérité.

En guise d'illustration, notons que Rolland analyse la notion de honte chez les personnages de Dostoïevski, en s'appuyant sur des remarques de Nietzsche puis de Levinas ${ }^{10}$. Rolland décèle chez ces personnages une «référence à une culpabilité antérieure à toute identifiable ou concrète - mais la honte qui en résulte ne tient pas tant ici à mon essence d'homme qu'à ma position de moi à la fois au monde et face à autrui. C'est seulement dans le mouvement d'aller vers les autres, d'entrer dans le rapport que se révèle une culpabilité préexistante et que dès lors jaillit la honte. Comme si, en présence d'autrui, mon évident droit à l'être se faisait question et comme si mon être-au-monde se révélait position en porte à faux»." Les thèmes de la honte liée à la culpabilité née de l'absence de légitimité de mon droit à être, qu'on retrouve explicitement chez Dostoïevski, ${ }^{12}$ sont sérieusement abordés par Levinas et Rolland en rend compte dans un certain nombre de textes qui servent de préfaces ou de notes à l'édition des textes dans lesquels Levinas traite ces sujets. ${ }^{13}$ De plus, les citations, parfois relativement longues de textes écrits par Levinas, sont récurrentes dans le livre de Rolland et régulièrement tout à fait intégrées aux développements de l'auteur. Par ailleurs, ce que Rolland tire de son analyse - et qu'il reformule en termes lévinassiens «antériorité et supériorité d'Autrui sur moi», «asymétrie» et «non-réciprocité», dimension essentiellement non-hégélienne ${ }^{14}$ - ancre définitivement la réflexion philosophique vers le problème de l'autre.

Le plan de l'ouvrage de Rolland s'articule autour de différentes modalités par lesquelles le moi est confronté, d'une certai-

10 Ibid., pp. 31-34.

11 Ibid., p. 32-33.

12 Voir Dostoïevski, 1994b, p. 348.

13 Par exemple l'introduction de L'éthique comme philosophie première, ou la préface à De l'évasion. Rolland définit cette culpabilité comme une «culpabilité sans faute - ou renvoyant à une circonstance... "ayant entraîné la mort sans l'intention de la donner"» (Rolland, 2000, p. 382).

14 Rolland, 1983, p. 33. 
ne façon, à l'autre. Les différentes caractérisations de la façon dont l'autre se manifeste pour les personnages de Dostoïevski sont autant de caractérisations de l'autre homme dans la philosophie de Levinas. Le premier moment examine «l'irruption d'autrui». Rolland montre que le roman dostoïevskien tel que le conçoit Bakhtine est un dialogue de personnages, et que ces personnages ont les attributs que Levinas confère au «moi» au début de Totalité et infini. ${ }^{15}$ Ces personnages occupent toujours des places socialement marquées dans l'univers dostoïevskien qui se caractérise par une forte hiérarchie sociale. Chaque individu poursuit ses buts en demeurant contraint de respecter le "réseau de convenances» ${ }^{16}$ qui sont comme les "remparts du même» (ce qu'illustre par exemple le fait de faire attendre Mychkine qui a l'air d'un vagabond, ou l'idée qu'on n'a pas à faire de cérémonie avec lui). En fonction de sa place dans la société hiérarchisée des romans de Dostoïevski, chacun, s'il cherche à persévérer dans son être, ne peut le faire que sous des formes autorisées par la société et peut - voire est encouragé à - traiter autrui en fonction de la distance qu'il croit le séparer de l'autre. Aussi, tant qu'un personnage respecte les convenances, il n'est pas directement confronté à autrui. Autrui se manifeste comme quelqu'un occupant une certaine place dans la société plus que comme altérité insaisissable. La place dans la société médiatise en quelque sorte le rapport à autrui et empêche le moi de faire face à l'altérité radicale d'autrui. Ce face à face survient lorsque le scandale, ${ }^{17}$

15 Ibid., p. 43. Comme l'écrit Rolland: «A un moi défini, convient un monde que parcourt, pour le diviser et l'unifier tout à la fois, une hiérarchie sociale. M'assignant une place en son sein, elle m'offre un lieu où peut, légitimement, s'exercer ma liberté - et les limites mêmes qu'elle impose à cette liberté en confirment la légitimité tout en en assurant la possibilité». Ce monde social fait d'autrui un Moi «Mais ainsi elle le dépouille de son altérité en l'insérant dans le monde qu'elle régit» (ibid., p. 44).

16 Ibid., p. 45.

17 On peut penser à Stavroguine au début des Possédés qui fait faire quelques pas à un vieillard en le tirant par le nez, à l'humiliation du capitaine Snegiriov par Dimitri Karamazov ou à Lisabeth Smerdiachtchaïa, fille de petit bourgeois, exemple de pauvreté et d'inconvenance, un peu folle, peu vêtue et quand on l'habille. Un nouveau gouverneur pense qu'elle «porte atteinte à la décence», les marchands l'habillent (Dostoïevski, 1994b, p. 110). Comme le note Rolland: «le véritable scandale [...] n'est pas provocation d'un moi exaspéré ne trouvant que dans la violence gratuite de quoi distraire son ennui - il est venue ou irruption d'Autrui. Dans ce monde mis en coupe, réglé, tapi derrière le rempart des convenances, 
brouillant les repères par lesquels le moi appréhende autrui, le rend imprévisible et incompréhensible pour le moi. C'est pourquoi d'après Rolland, le roman dostoïevskien n'est pas un roman social, mais un roman d'arrachement au social, ${ }^{18}$ signifiant par-là que le romancier ne s'en tient pas à une dénonciation de la société de son époque, mais qu'il veut mettre le moi à nu, creuser au-delà du social, parce que le social ne saurait dire le dernier mot, le mot le plus profond sur le moi. L'espace du roman ne serait plus alors l'espace social, mais un autre espace «ouvert que par l'irruption de l'Autre qui, comme le dit Rolland, venant comme d'outre-tombe, nous entraîne à passer le monde». ${ }^{19}$ Autrui ainsi révélé est souvent un personnage qui ne peut pas s'affirmer, dans le monde du roman de Dostoïevski, pleinement comme un moi. En effet, certains personnages forts de leur Moi ont une respectabilité sociale, comme les Epantchine ou la vieille princesse Belonskaïa. Mais cette respectabilité est inaccessible à d'autres personnages comme Mychkine, Nastassia Philippovna «relégués, par l'humiliation, la misère ou une native étrangeté dans une foncière abstraction, présentations de «l'homme dans l'homme», qui ne parviennent pas à se hausser jusqu'au statut de Moi». ${ }^{20}$ Le modèle de ces personnages en déficit de moi est, à n'en pas douter, Sonia dans Crime et Châtiment, qui apparaît dans le roman moins comme Sofia Marmeladova, qui serait un personnage mentionné selon l'état civil, socialement situé évoluant dans le monde, que comme, ainsi que le remarque Rolland, un «pur visage, visage porteur de "toute la douleur du monde"》, comme le lui dira un jour Raskolnikov. De ces personnages, il faudrait aller jusqu'à dire qu'ils ne sont pas du monde». ${ }^{21}$ Ainsi, à

\footnotetext{
Autrui survient. Et là est le scandale, car c'est dans la nudité d'être simplement un homme ou le premier venu qu'il survient sans consentir à la rassurante parure de la socialité, sans consentir à se faire «objet par son vêtement même» [citation de De l'existence à l'existant de Levinas], dans la fulgurance de qui ne m'approche pas par le biais d'une médiation, mais marrive de face» (Rolland, 1983, p. 48).

18 Ibid., p. 47.

19 Ibid.

20 Ibid., p. 49-50.

21 Ibid.
} 
première vue les grands romans de Dostoïevski s'inscrivent dans un monde social qui sépare et atomise les êtres tout en médiatisant leurs relations ; mais, comme le conclut J. Rolland

une lecture plus approfondie - ou une autre lecture - déplace son centre vers la description de la relation avec Autrui. Déplacement qui, dans le même temps, légitime l'existence de ce qui devient une périphérie sociale: la relation avec Autrui est relation entre un Moi et un Autre - et ce Moi séjourne dans un Monde. Ainsi, globalement, l'œuvre apparaît comme description de l'arrachement au monde - lequel se donne ici comme arrachement au réseau social hiérarchisé - arrachement que provoque l'irruption d'Autrui et arrachement vers l'autre espace, l'espace de la rencontre. ${ }^{22}$

Le second moment de l'analyse de Rolland rend compte de l'altérité en attribuant à l'Autre des caractéristiques également éminemment lévinassiennes: «Autrui, le Très-Faible et le Très-Haut». Dans cette partie, après avoir montré comment la nudité du visage permettait au moi d'entrer dans un face-à-face autrement que social avec autrui, l'auteur insiste sur la fragilité et la vulnérabilité d'autrui, ce que recouvre sa "pauvreté», tel qu'il m'apparaît dans ce face-à-face. Si autrui est pauvre, ce n'est évident pas seulement au sens socio-économique, et en comparaison avec des personnages dont, a contrario, on évoque la richesse. L'autre homme est pauvre absolument et pas seulement relativement. Comme l'écrit Rolland:

La pauvreté de l'homme battu, qui le montre comme le toujours exposé, le toujours sous la menace [...] est en effet essentielle en tant qu'elle l'atteint dans sa substance même, le prive de sa plénitude d'être. De sorte que la pauvreté, s'abstrayant de sa simple signification sociale, en vient à faire signe vers une misère plus profonde, que nous pouvons nommer Faiblesse. Faiblesse qui apparaît comme trait fondamental des personnages qui, dans les romans, interviennent comme figures ou figurations de l'Autre et qui s'y manifeste de diverses manières : par la misère économique d'un Marmeladov, l'humiliation d'un Ilioucha ou d'une Nastassia Philippovna, la maladie d'un Mychkine - toutes situation qui indiquent comme un moins-être. ${ }^{23}$

22 Ibid., p. 52.

23 Ibid., p. 54. 
La faiblesse ${ }^{24}$ ainsi déterminée peut être considérée comme «un moins-être, une impossibilité en quelque sorte à être, à être pleinement, à être sur le mode d'un Moi vivant dans la jouissance et se possédant dans une identité. Moins-être, non-être, termes inadéquats, qui ne parviennent qu'à assurer l'absolue préséance de l'être ${ }^{25}$ autrement dit, pour reprendre une formule chère à Levinas, un «autrement qu'être». Comme le premier moment de l'analyse permettait d'arracher autrui au monde socio-économique pour aller comme au-delà de lui, ce second moment détache autrui de l'ontologie, du domaine de l'être dans lequel chacun en est réduit à craindre pour sa vie. Le personnage dostoïevskien qui perçoit ainsi la faiblesse d'un autre personnage ne s'en tient pas à une compréhension théorique des choses, mais sent en lui la nécessité d'agir pour tenter - vainement - de combler ce manque d'être, n'agit plus seulement pour soi, n'est plus borné par la peur de sa propre mort ${ }^{26}$ comme quand Raskolnikov tente de sauver Marmeladov écrasé sous un attelage.

Ce troisième moment de l'analyse de la figure de l'altérité pour les personnages romanesques de Dostoïevski au prisme de la pensée de Levinas se donne comme passage de la responsabilité à la culpabilité. L'analyse par Rolland de cette idée insiste sur un point sur lequel on a souvent mal compris la pensée de Levinas. Dire, comme il le fait, que le moi est responsable d'autrui, c'est attirer l'attention sur la nudité et la misère de la chair, du corps exposé d'autrui, et c'est, par conséquent, marquer que «la responsabilité, d'emblée, conduit au don, au donner; don de son argent et de son temps, laissant déjà paraî-

24 Qui apparaît comme une caractéristique d'autrui dans une énumération de celles-ci (Levinas, 1997, p. 12).

25 Rolland, 1983, p. 57.

26 Comme en témoigne l'analyse, par Rolland, de la scène de Crime et châtiment où Svidrigaillov et Dounia sont décrits comme placés dans l'impossibilité de pouvoir sur l'autre. Svidrigaillov a attiré chez lui la sœur de Raskolnikov, pour lui révéler le crime de ce dernier et lui proposer de le sauver de la justice si elle se donne à lui. Après avoir refusé ce marché, Dounia tire sur lui et le manque. Dostoïevski parle du soulagement antinaturel de Svidrigaillov: ce dernier n'angoisse pas pour sa mort, mais craint pour autrui, crainte qu'il «ne devienne mon meurtrier. Et non pas à proprement parler mon meurtrier, mais celui de cet Autrui que je lui suis. [...] [car] s'il en vient à me tuer, ce sera - c'est déjà - ma faute» (ibid., p. 63-64). 
tre à l'horizon le don de soi. Vêtir ceux qui sont nus, ici, c'est peut-être soi-même se dévêtir». ${ }^{27}$ Dans Autrement qu'être, son maître ouvrage, Levinas soutient que la responsabilité va jusqu'à la substitution d'autrui, "prendre la place d'Autrui pour, finalement, mourir à sa place», ${ }^{28}$ comme l'explicite Rolland. Aussi, si l'auteur met-il en évidence que la notion de responsabilité conduit à culpabilité, grand thème des Frères Karamazov, il donne à la culpabilité ressentie par les personnages de ce roman une portée bien plus grande que la culpabilité œdipienne (celle que chaque ressentirait d'avoir prétendument toujours désiré tuer le Père) puisque la culpabilité dont il est question dans Les Frères Karamazov désignerait bien plutôt une «culpabilité envers les autres, tous les autres, et qui relève aussi d'une tout autre expérience». ${ }^{29}$

Le quatrième moment de l'analyse met au jour une autre caractéristique d'autrui: il est celui sur lequel le moi peut vouloir commettre un meurtre, et c'est ainsi qu'il apparaît aussi bien dans Totalité et infini de Levinas ${ }^{30}$ que dans Crime et châtiment. ${ }^{31}$ Autrui est celui que je peux vouloir tuer, et l'injonction éthique qui prend la forme de l'interdit du meurtre va jusqu'à l'exigence de tout faire pour empêcher que l'autre ne meure (ce qu'on a vu comme responsabilité et substitution). Or cette exigence éthique est lourde à porter et le héros des romans de Dostoïevski, à plusieurs reprises, tente de se défaire de cette responsabilité. Ainsi en est-il dans le passage de Crime et châtiment dans lequel Raskolnikov rencontre une toute jeune fille qu'on a enviré pour la violer, dont un autre homme semble vouloir également abuser. Raskolnikov veut défendre la jeune fille, se précipite sur l'homme et, quand arrive un policier, lui donne de l'argent et lui demande de protéger la malheureuse, avant de revenir sur ses pas pour conseiller au policier de lais-

27 Ibid., p. 77.

28 Ibid., p. 79.

29 Ibid., p. 80.

30 « Autrui est le seul être que je peux vouloir tuer » (Levinas, 1990, p. 216).

31 Rolland écrit ainsi, à partir de la scène ce l'aveu dans Crime et châtiment que «la raison du meurtre - sa raison et non son mobile - est à chercher dans la volonté d'un Moi de se poser absolument et, pour cela, de réduire l'altérité en tant que telle» (Rolland, 1983, p. 94). 
ser s'amuser l'autre homme car il pense que le policier et le gredin violeront la jeune fille. Raskolnikov dit alors: «Qu'est-ce que j'avais à vouloir venir à son secours, moi? Ah! bien, oui, secourir, est-ce à moi de le faire? Ils n'ont qu'à se dévorer les uns les autres tout vifs, que m'importe à moi». ${ }^{32}$ Commentant ce passage, Rolland écrit «Le problème qui se pose à Raskolnikov est le même que celui qui se pose à Ivan dans les Karamazov [...] : tenter de fuir cette responsabilité de secourir l'autre, le premier venu, cette responsabilité qui m'incombe à moi, hic et nunc». ${ }^{33}$

Le cinquième moment étudie l'impossibilité de répondre à l'exigence éthique de responsabilité allant jusqu'à la substitution, à laquelle nous soumet le face-à-face avec autrui. En effet, s'il est possible d'éluder la tentation du meurtre et de porter secours autant que nous le pouvons à autrui au spectacle de la détresse, de sa misère nue et de sa faiblesse foncière - ce que Levinas, parfois, appelle du nom de «Réveil» - la sainteté ne peut pas être un état auquel on atteindrait d'une façon ou d'une autre. Comme l'écrit Rolland: «Éveil qui ne se sédimente pas, ne se substantifie pas en vertu et ne conduit pas à l'état de sainteté où le Moi déjà s'endormirait ou «s'embourgeoiserait». Dénonciation de l'idée même de sainteté comme état». ${ }^{34}$ L'éveil est comme la suspension ou l'interruption éthique du conatus spontané et naturel, par lequel chaque être tend à persévérer dans son être. Il peut arriver que ponctuellement, provisoirement, momentanément, on parvienne à ne pas se dés-inter-esser de l'autre et qu'on s'éveille parce qu'on est responsable pour lui, mais cette responsabilité n'est pas éternelle, et tôt ou tard, la suspension ou l'interruption éthique se termine. Cela confirme autant l'irréductibilité de l'altérité à l'identité que la démesure de l'exigence éthique à laquelle

32 Dostoïevski, 1995, p. 91.

33 Rolland, 1983, p. 97.

34 Ibid., p106. Cf. «La vigilance - réveil dans l'éveil - signifie la dé-fection de l'identité, ce qui n'est pas son extinction mais sa substitution au prochain - ordre ou désordre de la raison n'est plus ni connaissance ni action mais où désarçonnée par Autrui de son état désarçonnée du Même et de l'être - elle est en relation éthique avec autrui, proximité du prochain.»(Levinas, 1998b, p. 60). 
me confronte la fragilité d'autrui, à laquelle je ne puis jamais satisfaire. ${ }^{35}$ C'est pourquoi moins que d'un échec de la réponse éthique à l'appel de la faiblesse d'autrui, Rolland préfère parler d'une «tension». ${ }^{36}$

\section{Par-delà Levinas et Bakhtine : l'altérité du Christ et la lecture de Catteau}

Si la caractérisation des figures de l'altérité telle qu'elle se manifeste dans l'univers romanesque dostoïevskien peut être majoritairement décrite en termes empruntés à la philosophie de Levinas, il reste une figure de l'altérité propre au romancier russe qui n'est pas intégrée à la pensée du philosophe: c'est la figure du Christ, figure capitale de la littérature russe de cette époque et incontournable ${ }^{37}$ pour rendre compte des romans de Dostoïevski. Aussi Rolland présente-t-il comme un «rebondissement», l'analyse qu'il donne de la figure de l'altérité qu'est le Christ dans les romans dostoïevskiens, dans la mesure où cette perspective déborde la reformulation en termes lévinassiens de cette altérité. L'analyse de J. Rolland toutefois ne comptabilise pas cette présence du Christ simplement comme un addendum, un supplément sans grande portée, mais défend la thèse qu'elle change le cœur du roman de Dostoïevski, au point d'aboutir à l'«hypothèse sur le sens profond du roman dostoïevskien en tant que roman religieux». ${ }^{38}$

\footnotetext{
35 Rolland insiste sur cette exigence éthique démesurée, que Levinas incline «dans une direction particulière, et indéniablement inquiétante. [...] La responsabilité est tout aussi in-finie qu'elle est impossible: encore responsable d'un retard qu'elle ne saurait combler» (Rolland, 2000, p. 384).
}

36 «Tension entre l'impossibilité d'une réponse et sa nécessité ou son urgence. Mais tension dans laquelle, malgré l'impossibilité de la réponse, un appel persiste et maintient son appel, tension où se met en question la réduction de l'humain à la possibilité, à l'initiative et à la liberté. Tension dans laquelle se lève peut-être dès lors un sens nouveau - ou très ancien de l'homme en sa condition ou son in-condition de sujet responsable de tout, c'est-à-dire encore de ce qu'il n'a pas voulu et de ce dont il ne peut pas répondre» (Rolland, 1983, p. 115).

37 «La remise en jeu du questionnement tient à l'impossibilité d'ignorer l'inscription dans l'œuvre d'un pôle d'altérité non humaine sous les espèces de la figure du Christ», ibid., p. 123.

38 Ibid., p. 125. 
Qu'apporte la figure du Christ à la figuration de l'altérité? On perd, par rapport à ce qui avait été dit de l'altérité deux éléments selon Rolland; d'une part «le caractère absolument premier du rapport d'altérité que rien ne conditionne mais qui en lui-même conditionne jusqu'à la possibilité de signifiance de $l^{\prime}$ Infini $»^{39}$ et «le caractère absolument immédiat de cette relation telle que le face à face qui la constitue constitue encore le paradigme même de toute immédiateté»..$^{40} \mathrm{En}$ effet, ce qui change avec la figure du Christ c'est d'une part qu'il est une figure de la médiation: la Faiblesse de l'autre, d'une certaine façon, et son appel ne valent plus pour eux-mêmes, mais pour l'amour du Christ. Et même, cette médiation finit par annuler la question de l'Autre en tant que telle, puisqu'avec le Christ ce qui est visé n'est plus «le respect de l'Autre en son altérité, le maintien de cette altérité et par là le maintien [...] d'une relation asymétrique mais, tout au contraire, sa réduction et sa dissolution dans et par la fusion des termes dans laquelle s'ouvre la voie du salut», ${ }^{41}$ comme le note Rolland. Et l'auteur illustre ce changement de perspective, entre une conception de l'altérité sans le Christ à une conception de l'altérité avec le Christ, par l'examen du personnage important qu'est Sonia. Cette dernière est une figure centrale en tant que visage qui adresse un appel dans la perspective inspirée de Levinas, mais dans le cadre d'un rapport au Christ, Sonia est une

figure sans doute toujours essentielle, mais non plus centrale à proprement parler, lorsqu'elle se fait messagère qui, par son statut même, s'efface devant ce qu'elle annonce comme le signe devant ce qu'il signale. Ce qu'ainsi elle annonce, c'est la Parole évangélique par laquelle le Christ offre cette promesse en léguant sa Parole. [...] dans cet effacement, elle cède à la figure du Christ la place de figure centrale ru roman, figure sans doute elle-même absente du roman - mais roman qui ne s'écrit cependant que pour l'inscrire au bout de lui-même, où se dresse sa Hauteur et s'exalte sa Parole. ${ }^{42}$

39 Ibid., p. 127.

$40 \mathrm{lbid}$.

41 Ibid., p. 129.

42 Ibid., p. 138. 
Si Rolland semble amender sa lecture lévinassienne de l'altérité dans les romans de Dostoïevski en faisant saillir à la fin de l'analyse la présence du Christ inintégrable dans la perspective du philosophe, il veut relativiser et compléter la thèse de Bakhtine qu'il avait jusqu'ici reprise à son compte sur la nature du roman dostoïevskien

en réintroduisant la dimension de l'auteur, ${ }^{43}$ c'est-à-dire en n'oubliant pas, ce que Bakhtine a trop tendance à faire, que si Dostoïevski innove en créant un espace polyphonique dans lequel le personnage a sa pleine autonomie, il en est par définition le créateur dont la création relève d'un projet que caractérise un certain vouloir-dire. ${ }^{44}$

Il s'appuie, pour cela, sur le travail de Catteau sur la comparaison entre les états préparatoires et le texte définitif des romans. En effet, selon Catteau, à la lecture des premières versions, le romancier demeure "pleinement souverain»: «Dostoïevski y apparaît comme l'ordonnateur suprême des gestes et le maitre des vies [...] comme tout romancier, il façonne les hommes, ses héros, avec une volonté de dire, et même parfois de transmettre un message». ${ }^{45}$ Aussi, contrairement à ce que soutient Bakhtine, le projet littéraire de l'auteur conditionne la relative autonomie des personnages: ils sont libres, mais dans le cadre décidé par l'auteur. L'idée qui prévalait depuis le début des analyses de Rolland, selon laquelle le rapport d'altérité constitue, en se déployant, le roman dostoïevskien, apparaît ainsi comme corrigée. Et Rolland conclut que «la caractérisation formelle du roman dostoïevskien, retenant la leçon de Bakhtine mais refusant de se laisser enfermer dans le dogmatisme dont il n'est pas toujours indemne, le fait apparaître comme une structure non identitaire: structure animée par une tension, tension entre homophonie et polyphonie, entre fermeture et ouverture, achèvement et inachèvement, Livre et écriture». ${ }^{46}$

43 Pour Todorov, la conception bakhtinienne du roman de Dostoïevski fait des personnages des quasi auteurs «les personnages de Dostoïevski [...] sont comme autant d'auteurs, plutôt que comme les personnages des anciens auteurs», Todorov, 1981, p. 159).

44 Rolland, 1983, p. 141.

45 Ibid., p. 142.

46 Ibid., p. 147. 


\section{Conclusion}

La façon qu'a Rolland se formuler en termes lévinassiens l'altérité dans les romans de Dostoïevski est très pertinente et fait preuve d'une grande familiarité avec la philosophie lévinassienne, mais elle en reste majoritairement à la perspective d'avant Autrement qu'être. Rolland fait ressortir l'incomplétude, le manque d'être de certains personnages, mais ne va pas jusqu'à faire voir la passivité originaire du sujet (que des personnages comme Aliocha ou Mychkine ne seraient pas loin de pouvoir figurer). En effet, on pourrait dire qu'avant Autrement qu'être, la passivité liée au visage d'autrui brisait le sujet, faisait éclater l'égoïté dans l'irruption de l'autre. Mais avec $A u-$ trement qu'être, Levinas envisage «le sujet comme passivité en son origine même [...] Passivité d'un sujet non plus pensé comme le Même déjà constitué qui ensuite rencontre l'Autre, et avec lui la limite de son pouvoir - mais, originairement, structuré comme Autre-dans-le-Même.» ${ }^{47}$

De plus, s'il est tout à fait pertinent de réfléchir à la façon dont Bakhtine pense le roman dostoïevskien comme polyphonique, cette approche reste peut-être en deçà de la pensée bakhtinienne de l'altérité. En effet, comme l'écrit Todorov dans un ouvrage cité par Rolland, pour Bakhtine, l'être humain «n'existe qu'en dialogue: au sein de l'être on trouve l'autre» ${ }^{48}$ ou «pour Bakhtine, «au fond de l'homme», n'est pas le «ça», mais l'autre».49

47 Rolland, 2000, p. 384.

48 Todorov, 1981, p. 9

49 Ibid., p. 55. Voir également, sur l'importance d'autrui et le fait que je ne suis par l'intermédiaire d'autrui, ainsi que sur le rôle d'autrui dans la prise de conscience de qui je suis, les pages 148 à 150 du même ouvrage, en particulier: «Je ne deviens conscient de moi, je ne deviens moi-même qu'en me révélant pour autrui, à travers autrui et à l'aide d'autrui. Les actes les plus importants, constitutifs de la conscience de soi, se déterminent par le rapport à une autre conscience (à un «tu»). La rupture, l'isolement, l'enfermement en soi est la raison fondamentale de la perte de soi. [... Toute expérience intérieure s'avère être située à la frontière, elle rencontre autrui, et toute son essence réside dans cette rencontre intense. [...] L'être même de l'homme (extérieur comme intérieur) est une communication profonde. Être signifie communiquer. [...] être signifie être pour autrui et, à travers lui, pour soi». 


\section{Références}

BAKHTINE, Mikhaïl. La Poétique de Dostö̈evski, traduit par Isabelle Kolitcheff. Paris : Seuil, 1970.

BERDIAEV, Nicolas. L'Esprit de Dostoïevski, traduit par Alexis Nerville. Paris: Stock, 1974.

CATTEAU, Jacques. La Création littéraire chez Dostoïevski. Paris : Institut d'Études slaves, 1978.

DOSTOÏEVSKI, Fédor. Crime et Châtiment, traduit par D. Ergaz. Paris: Gallimard, 1995.

DOSTOÏEVSKI , Fédor. L'Idiot, traduit par Albert Mousset. Paris : Gallimard, 1994a.

DOSTOÏEVSKI, Fédor. Les Possédés, traduit par Elisabeth Guertik. Paris: Le livre de poche, 1977.

DOSTOÏEVSKI, Fédor. Les Frères Karamazov, traduit par Elisabeth Guertik. Paris: Le livre de poche, 1994b.

LEVINAS, Emmanuel. De l'évasion, éd. annotée par J. Rolland. Paris: Le livre de poche, 1998a.

LEVINAS, Emmanuel. De l'existence à l'existant. Paris : Vrin, 1947.

LEVINAS, Emmanuel. Totalité et infini : essai sur l'extériorité, Paris: Le livre de poche, 1990.

LEVINAS, Emmanuel. Autrement qu'être ou au-delà de l'essence. Paris: Le livre de poche, 2001.

LEVINAS, Emmanuel. De Dieu qui vient à l'idée. Paris: Vrin, 1998b.

LEVINAS, Emmanuel. Hors sujet. Paris: Le livre de poche, 1997.

LEVINAS, Emmanuel. L'Éthique comme philosophie première, introduction de J. Rolland. Paris: Payot \& Rivages, 1998c.

ROLLAND, Jacques. Dostoïevski. La Question de l'autre. Paris: Verdier, 1983.

ROLLAND, Jacques. Parcours de l'autrement: lecture d'Emmanuel Levinas. Paris: PUF, 2000.

TODOROV, Tzvetan. Mikhaïl Bakhtine le principe dialogique. 
Suivi de Écrits du Cercle de Bakhtine. Paris : Seuil, 1981.

Recebido em: 04/09/2021

Aceito em: 30/11/2021 